\title{
Le référendum britannique du 23 juin 2016 : un simulacre de démocratie?
}

The British Referendum of June 23 2016: a Mockery of Democracy?

\section{Christian Auer}

\section{CpenEdition}

\section{Journals}

Édition électronique

URL : http://journals.openedition.org/rfcb/1337

DOI : $10.4000 /$ rfcb. 1337

ISSN : 2429-4373

Éditeur

CRECIB - Centre de recherche et d'études en civilisation britannique

Référence électronique

Christian Auer, "Le référendum britannique du 23 juin 2016 : un simulacre de démocratie? 》, Revue Française de Civilisation Britannique [En ligne], XXII-2 | 2017, mis en ligne le 30 mai 2017, consulté le 20 avril 2019. URL : http://journals.openedition.org/rfcb/1337 ; DOI : 10.4000/rfcb.1337

Ce document a été généré automatiquement le 20 avril 2019

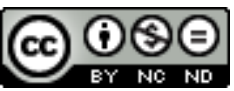

Revue française de civilisation britannique est mis à disposition selon les termes de la licence Creative Commons Attribution - Pas d'Utilisation Commerciale - Pas de Modification 4.0 International. 


\title{
Le référendum britannique du 23 juin 2016 : un simulacre de démocratie?
}

The British Referendum of June 23 2016: a Mockery of Democracy?

\author{
Christian Auer
}

\section{Introduction}

1 Lors du référendum du 23 juin 2016 sur le maintien du Royaume-Uni dans l'Union européenne, les Britanniques ont été amenés à prendre une décision fondamentale non seulement pour l'avenir de leur pays, mais aussi pour l'Union toute entière. A la question "Should the United Kingdom remain a member of the European Union or leave the European Union?», 51,9\% des Britanniques ont répondu qu'ils souhaitaient sortir de l'Union, précipitant ainsi leur pays et l'Union dans l'instabilité et l'incertitude. La victoire des partisans du Leave rendra plus difficile l'élargissement de l'Union et le renforcement de la coopération en matière de défense, de politique étrangère ou environnementale et pourrait inciter d'autres pays à entreprendre une démarche similaire. Le sentiment antieuropéen existant dans certains pays, qui se matérialise notamment par la percée spectaculaire des partis nationalistes anti-européens ou eurosceptiques, pourrait être amené à se développer encore davantage. Les revendications séparatistes, comme en Ecosse, en Catalogne, au Pays basque ou en Italie du Nord, pourraient gagner en puissance.

D'après un rapport établi par Global Counsel ${ }^{1}$, structure créée en 2010 par un groupe d'experts britanniques et européens, les trois pays les plus exposés à une sortie du Royaume-Uni de l'Union européenne seraient les Pays Bas, Chypre et l'Irlande. Les investissements des entreprises néerlandaises au Royaume-Uni représentaient près de $1,5 \%$ du PNB néerlandais en 2013. Les liens entre les banques néerlandaises et britanniques sont très importants, les prêts accordés par les banques britanniques se montant à 236 milliards d'euros en 2014. De surcroît, les Pays Bas partagent des positions 
très proches de celles du Royaume-Uni, les deux pays militant pour une diminution des régulations du marché et une ouverture du commerce extérieur. La République de Chypre sera, elle-aussi, directement touchée par la sortie du Royaume-Uni de l'Union européenne : les deux pays partagent une histoire et une culture commune. Chypre est également membre du Commonwealth et héberge deux bases militaires britanniques. Près de trois cent mille personnes d'origine chypriote vivent au Royaume-Uni, ce qui représente un tiers de la population actuelle de Chypre. Pour des raisons historiques évidentes, les rapports entre l'Irlande et le Royaume-Uni sont extrêmement forts. Les exportations irlandaises de biens et de services vers le Royaume-Uni en 2013 ont représenté près de $12 \%$ du PNB irlandais, un chiffre beaucoup plus important que la moyenne des autres pays de l'Union. Les deux pays partagent, en outre, une idéologie économique libérale, ce qui en fait des partenaires privilégiés au sein de l'Union. Toutes ces tensions, encore exacerbées en raison des multiples difficultés auxquelles l'Europe doit actuellement faire face, pourraient porter un coup fatal à une Union qui éprouve des difficultés à conserver son identité, pour peu que cette identité ait jamais existé.

Cet article aura comme ambition de démontrer que les citoyens britanniques furent dans l'impossibilité de faire un choix éclairé dans la mesure où les spécialistes les plus éminents proposèrent des scénarios économiques différents, voire totalement contradictoires. Le référendum sur le Brexit, qui tirait avant tout son origine du souhait de David Cameron de donner satisfaction à la frange la plus eurosceptique de son parti, tendit à faire croire aux citoyens britanniques qu'ils avaient les capacités et les compétences pour répondre à une question, certes formulée de façon claire, comme il est de coutume dans un exercice de démocratie directe de ce type, mais en fait éminemment complexe. Cet article conclura en remarquant que l'exercice référendaire peut avoir toute sa pertinence quand sont posées des questions d'ordre moral ou éthique, où les citoyens sont en mesure de juger par eux-mêmes, mais semble inapproprié quand ils sont amenés à se prononcer sur des questions techniques d'ordre constitutionnel ou économique pour lesquelles des connaissances ou des compétences spécifiques sont requises, voire indispensables.

\section{Les référendums au Royaume-Uni}

Depuis 1973, onze référendums ont eu lieu au Royaume-Uni. La plupart de ces consultations concernaient le transfert de pouvoir à des assemblées ou parlements régionaux ou nationaux. Les référendums du 8 mars 1973 et du 22 mai 1998 concernaient la seule Irlande du Nord, ceux des $1^{\text {er }}$ mars 1979, 11 septembre 1997 et du 18 septembre 2014 l'Ecosse, ceux des $1^{\text {er }}$ mars 1979, 18 septembre 1997 et du 3 mars 2011 le Pays de Galles et celui du 7 mai 1998 la ville de Londres. Seuls deux référendums ont porté sur des questions concernant l'ensemble des citoyens du Royaume-Uni, celui du 5 juin 1975 sur le maintien du Royaume-Uni au sein de la Communauté Européenne ${ }^{2}$ et celui du 5 mai 2011 où il fut demandé aux Britanniques s'ils souhaitaient changer le système électoral pour les élections des députés à la Chambre des Communes ${ }^{3}$. L'outil de démocratie participative que constitue le référendum a donc été très peu utilisé pour des questions concernant le Royaume-Uni dans son ensemble.

Dans l'architecture constitutionnelle britannique, la suprématie du Parlement est absolue et figure au nombre des "doctrines sacrosaintes $»^{4}$, ce qui explique les nombreuses réticences exprimées à l'encontre de cette pratique. Comme l'a souligné Geoffrey 
Marshall :

The referendum device has not on the whole been well-regarded in British political discourse. [...] by and large, most publicists and politicians, whether of liberal or conservative persuasion, have been anti-referendum ${ }^{5}$

Ce commentaire mérite, toutefois, d'être nuancé car l'idée du recours au référendum fut évoquée par de nombreux hommes politiques britanniques au cours de l'histoire. On se contentera de citer Joseph Chamberlain, Arthur Balfour, Lord Curzon, Winston Churchill qui tous, à un moment ou à un autre, envisagèrent de recourir au référendum, soit pour résoudre des questions économiques ou constitutionnelles, soit pour trouver une issue à des impasses ou blocages politiques, comme ce fut le cas en 1909 quand Arthur Balfour n'exclut pas de recourir au référendum pour mettre un terme au conflit qui opposait les deux Chambres du Parlement :

If you ask me whether this constitutional machinery could not be improved, either by some change in the composition of the House of Lords, or by the institution of a Referendum, I am certainly not going even to suggest a negative reply.

6 Autre exemple, avec Margaret Thatcher qui, lorsqu'elle était leader de l'opposition, considéra qu'un référendum pourrait s'avérer utile afin de résoudre le problème de la toute puissance des syndicats. C'est ce qu'elle indiqua pendant le conflit qui, entre 1976 et 1978, opposa les syndicats aux dirigeants d'une entreprise située à Londres, conflit devenu célèbre sous le nom de "Grunswick dispute»: "I argued that although such a confrontation was unlikely, yet if such an emergency was reached, then a referendum might be necessary ${ }^{7} »$. On ajoutera que la position de Margaret Thatcher sur la pratique référendaire ne fut pas toujours dénuée d'ambiguïté: ainsi, lors des débats sur le référendum de 1975 concernant le maintien du Royaume-Uni au sein de la Communauté européenne, elle cita Clement Attlee qui avait indiqué que le référendum était une mesure à laquelle avaient recours les dictateurs et les démagogues ${ }^{8}$.

7 Les opinions sur l'utilisation du référendum sont donc extrêmement variées et le plus souvent tranchées. Cela ne saurait surprendre tant cette pratique et son instrumentalisation peuvent représenter un risque de tyrannie de la majorité et d'affaiblissement des gouvernements et de la souveraineté parlementaire. Le cas du référendum du 5 juin 1975 sur le maintien du Royaume-Uni au sein de la Communauté économique européenne (CEE) est particulièrement intéressant car il donna lieu à de multiples débats sur la nature et le bien-fondé de la pratique référendaire. Ainsi, lors du débat qui se tint à la Chambre des Communes le 11 mars 1975, Margaret Thatcher exprima avec beaucoup de pertinence les difficultés inhérentes à une démarche dans un pays pour lequel la notion de souveraineté parlementaire constitue une des pierres angulaires du système constitutionnel :

To use the referendum device at all is to ask the question: to what category of measure should referenda apply? Presumably the answer would be: in cases of constitutional change. But it is hard to define such a change in the British tradition because so much depends on convention and precedent. A referendum may, however, become acceptable if given a proper constitutional foundation - that is to say, if the conditions under which it could be used were defined. But that would mean, like many other democratic countries, going as far as a written constitution or at least part of the way. The implications for parliamentary sovereignty are profound. But if our sense of constitutional rules and conventions is weakening, there may come a time when some such course should be considered. ${ }^{9}$ 


\section{Une Union européenne réformée?}

8 Il convient de rappeler que le vote du 23 juin 2016 devait aussi permettre aux Britanniques de se prononcer sur la nature des garanties et des modifications obtenues par le Premier ministre lors de ses négociations avec l'Union européenne. David Cameron s'était en effet engagé à obtenir un statut spécial pour le Royaume-Uni au sein de l'UE. Le pari était risqué et rappelle étrangement la stratégie à laquelle eut recours le parti travailliste en 1974 pour convaincre les électeurs de voter en faveur du maintien du Royaume-Uni au sein de la Communauté économique européenne. Harold Wilson s'était en effet engagé à obtenir de meilleures conditions que celles obtenues par Edward Heath lors des négociations avec la CEE. Si le Royaume-Uni obtenait satisfaction le gouvernement se prononcerait pour le oui au référendum. En cas d'échec des négociations, Wilson soutiendrait le camp du non:

Britain is a European nation, and a Labour Britain would always seek a wider co-operation between the European peoples. But a profound political mistake made by the Heath Government was to accept the terms of entry to the Common Market, and to take us in without the consent of the British people. [...]'If re-negotiations are successful, it is the policy of the Labour Party that, in view of the unique importance of the decision, the people should have the right to decide the issue through a General Election or a Consultative Referendum. If these two tests are passed, a successful renegotiation and the expressed approval of the majority of the British people, then we shall be ready to play our full part in developing a new and wider Europe. 'If re-negotiations do not succeed, we shall not regard the Treaty obligations as binding upon us. We shall then put to the British people the reasons why we find the new terms unacceptable, and consult them on the advisability of negotiating our withdrawal from the Communities.' 10

Wilson, estimant que les négociations avec ses partenaires européens avaient été couronnées de succès, s'engagea en faveur du maintien du Royaume-Uni dans la Communauté économique européenne en dépit des profondes réserves exprimées par certains des membres de son Cabinet, au nombre desquels Tony Benn ne fut pas le moins virulent: "The Community will destroy the whole basis on which the labour movement was founded and its commitment to democratic change " ${ }^{11}$.

10 Comme nous le savons et malheureusement pour David Cameron, l'histoire ne s'est pas répétée. Les grandes lignes des revendications formulées par le Premier ministre figurent dans une lettre datée du 10 novembre 2015 qu'il fit parvenir à Donald Tusk, le président du Conseil européen. Comme l'indiqua clairement David Cameron, le maitre mot était flexibilité : «Our concerns really boil down to one word: flexibility $»^{12}$. La lettre comportait quatre points distincts, la gouvernance économique, la compétitivité, la souveraineté et l'immigration. Après de multiples discussions et négociations, un accord ${ }^{13}$ fut finalement trouvé entre les 28 pays membres le 19 février 2016, ce qui permit à David Cameron d'annoncer qu'il avait obtenu des garanties suffisantes pour recommander le maintien de son pays dans l'Union. Il suffit, cependant, d'examiner les termes de l'accord pour se rendre compte qu'il est difficile d'évaluer avec précision les avancées réelles obtenues par David Cameron. M. Cameron n'a pas obtenu satisfaction pour ce qui est de la question des allocations familiales attribuées aux migrants européens. Devant la forte opposition de certains pays, dont la Pologne, il dut revoir ses exigences à la baisse : même si le montant des sommes allouées aurait été fonction du niveau de vie du pays concerné, les travailleurs migrants auraient pu continuer à envoyer une partie du montant de leurs 
allocations dans leurs pays d'origine. Il semblerait que $\mathrm{M}$ Cameron ait obtenu partiellement gain de cause en ce qui concerne la monnaie européenne : l'euro ne serait pas considéré comme étant la seule monnaie de l'Union. Pour ce qui est de la question de la souveraineté nationale, le Premier ministre avait exprimé ses revendications dans les termes suivants :

I want to end Britain's obligation to work towards an "ever closer union" as set out in the Treaty. It is very important to make clear that this commitment will no longer apply to the United Kingdom. I want to do this in a formal, legally-binding and irreversible way. ${ }^{14}$

11 Il convient tout d'abord de remarquer que les termes "d'union de plus en plus étroite » sont excessivement difficiles à cerner avec précision. Comme l'indiqua la commission de la London School of Economics chargée de réfléchir à l'avenir du Royaume-Uni au sein de l'Union, le concept, qu'elle qualifie d'ambigu et d'énigmatique, est de nature politique et peut donner lieu à des interprétations différentes, voire divergentes ${ }^{15}$. Les conclusions des négociations concernant ce point particulier furent donc loin de représenter une victoire pour David Cameron : «It is recognised that the United Kingdom, in the light of the specific situation it has under the Treaties, is not committed to further political integration into the European Union $»^{16}$. En effet, le fait que le Royaume-Uni s'engage ou non en faveur du principe "d'une union de plus ou plus étroite » n'aurait eu aucune incidence sur la nature de l'évolution de l'Union. Pour ce qui est de la demande la moins controversée du Premier ministre, à savoir la question de la compétitivité, M Cameron se targua d'avoir obtenu des résultats dépassant ses espérances. L'Union européenne s'était en effet explicitement engagée à réduire les contraintes administratives pesant sur les citoyens et les entreprises :

The European Parliament, the Council and the Commission have agreed the Interinstitutional Agreement on Better Law Making. Effective cooperation in this framework is necessary in order to simplify Union legislation and to avoid overregulation and administrative burdens for citizens, administrations and businesses, including small and medium sized enterprises, while ensuring that the objectives of the legislation are met. ${ }^{17}$

Il est clair, cependant, que de tels engagements sont suffisamment vagues pour ne pas être contraignants. On remarquera, de surcroit, que les instances européennes ont par le passé manifesté des ambitions similaires qui n'ont été suivies d'aucun effet. Lors d'un récent débat sur l'avenir de l'Europe à Rome, en présence du président de la Commission européenne Jean-Claude Juncker, des présidents du Conseil européen Donald Tusk et du Conseil italien Matteo Renzi, le président du Parlement européen Martin Schulz, reconnaissait lui-même que l'Europe était une promesse qui n'avait pas été tenue ${ }^{18}$.

Ces quelques remarques prouvent que l'étendue réelle des garanties obtenues par le Premier ministre est difficile à évaluer, surtout pour des citoyens peu habitués à décoder la rhétorique des institutions européennes. Cette question était pourtant essentielle car si les citoyens britanniques étaient censés se prononcer sur le maintien de leur pays dans une Europe " réformée ", encore fallait-il qu'ils fussent en mesure de comprendre en quoi ces réformes modifieraient la structure et le fonctionnement de l'Union. Il y eut donc clairement deux aspects distincts mais interconnectés dans la décision que furent amenés à prendre les Britanniques. Avant même d'envisager de répondre à la question du maintien du Royaume-Uni dans l'Union, question formulée de façon très simple en apparence, le citoyen était censé au préalable répondre à une autre question sous jacente: l'Union européenne, telle que présentée, est-elle réformée ou non ? Sans réponse claire à 
cette première question, les Britanniques étaient dans l'incapacité d'apporter une réponse éclairée à la question qui leur était posée.

\section{Les enjeux du référendum} souveraineté, l'immigration, la place du Royaume-Uni dans le monde et la sécurité figurèrent au centre des débats. La législation européenne stipule que les Britanniques ont la possibilité de vivre et de travailler dans tout pays de l'Union. Ces règles s'appliquent bien évidemment aux citoyens de l'Union qui peuvent venir s'établir et travailler au Royaume-Uni. Il en a résulté une augmentation importante de l'immigration au Royaume-Uni notamment en provenance des pays de l'est et du sud de l'Europe. Les partisans du maintien du Royaume-Uni dans l'Union reconnurent que la montée de l'immigration engendrait un certain nombre de difficultés, notamment dans le secteur du logement, mais indiquèrent que l'immigration avait des effets bénéfiques dans l'ensemble. Pour David Cameron, les concessions obtenues lors de ses négociations avec l'Union devaient permettre de réduire l'immigration vers le Royaume-Uni. Les partisans d'une sortie de l'Union insistèrent, au contraire, sur le fait que la forte augmentation de l'immigration représentait une menace pour la cohésion sociale et que le Brexit permettrait au Royaume-Uni de reprendre le contrôle de ses frontières et donc de réduire l'immigration de façon significative.

Pour ce qui est de la place du Royaume-Uni dans le monde, les avis divergeaient radicalement. Les partisans du maintien considéraient qu'en cas de Brexit le Royaume-Uni perdrait de son influence sur la scène internationale; les Etats-Unis pourraient aussi s'éloigner de son allié historique et se rapprocher de partenaires qui conserveraient une certaine influence internationale. Les partisans du Brexit firent remarquer qu'une sortie n'aurait aucune incidence sur la place du Royaume-Uni dans le monde. Autre point de clivage entre les deux camps, la sécurité. Iain Duncan Smith, l'ancien ministre du Travail, qui rejoignit le camp du Brexit, indiqua que le maintien dans l'Union augmentait les risques d'attentats :

This open border does not allow us to check and control people that may come and spend time [...] We've seen what happened in Paris where they spent ages planning and plotting so who's to say it's not beyond the wit of man that those might already be thinking about that. ${ }^{19}$

Cette position, qui résumait les inquiétudes des partisans du Brexit, fut partagée par certains spécialistes du terrorisme international. Ce fut le cas notamment de Richard Kemp, ancien colonel de l'armée britannique, qui indiqua qu'une sortie de l'Union permettrait au Royaume-Uni de reprendre le contrôle de ses frontières. D'après lui, la situation actuelle augmentait le risque d'attaque terroriste et constituait une trahison de la nation britannique. Les partisans du maintien, à l'image d'anciens responsables de la sécurité comme Lord Bramall et Jock Stirrup, rejetaient en bloc ces arguments et remarquaient que faire partie de l'Union constituait un avantage indiscutable dans un monde de plus en plus instable. Michael Fallon, le ministre de la défense du gouvernent britannique, insista, quant à lui, sur les autres avantages de l'Union:

It is through the EU that you exchange criminal records and passenger records and work together on counter-terrorism [...] We need the collective weight of the EU when you are dealing with Russian aggression or terrorism ${ }^{20}$.

Revue Française de Civilisation Britannique, XXII-2 | 2017 


\section{Les enjeux économiques}

17 Les aspects d'ordre économique constituèrent sans nul doute les enjeux majeurs du référendum. Les Britanniques furent en effet amenés à se déterminer par rapport à des questions aussi complexes que le commerce, l'investissement, le marché de l'emploi ou encore la libre circulation des biens et des capitaux. L'examen des arguments principaux proposés par les deux camps permettra de se rendre compte de l'étendue de la complexité de la question. L'organisation officielle du camp du Remain, désignée par la Electoral Commission, Britain Stronger in Europe, créée le 12 octobre 2015, publia un document de 35 pages en réponse aux arguments développés par le camp des partisans de la sortie de l'Europe. Le document précise que trois millions d'emplois britanniques dépendent directement du commerce avec les pays de l'Europe, que 200000 entreprises ont des relations commerciales avec des entreprises européennes, ce qui représente $75 \%$ du commerce britannique, que l'Europe absorbe la moitié des exportations britanniques et que le Royaume-Uni perçoit 66 millions de livres de l'Europe chaque jour. Le document présente ensuite 100 points, émanant des figures les plus influentes et compétentes du secteur économique, censés prouver l'absurdité des thèses des tenants de la sortie de l'Union. Il serait quelque peu fastidieux d'en donner la liste exhaustive mais les quelques exemples qui suivent permettent non seulement de saisir la grande diversité des questions que soulevait le référendum mais aussi de se rendre compte de la quasi impossibilité pour le non spécialiste en économie (ce qui est le cas de la très grande majorité des citoyens, qu'ils soient britanniques ou non) de prendre une décision éclairée.

L'accès au marché unique européen pourrait apporter 60 milliards de livres à l'économie britannique et créer 800000 emplois d'ici 2030. Une sortie de l'Union, par contre, entraînerait une dévaluation de la livre pouvant atteindre $20 \%$ et contraindrait le Royaume-Uni à négocier plus d'une cinquantaine d'accords bilatéraux en remplacement des accords existants, diminuant considérablement l'influence de l'économie britannique. De surcroit, l'inflation augmenterait fortement, le marché du logement pourrait connaître une période de récession et le prix de l'essence augmenterait de près de $20 \%$. Le gouvernement britannique serait contraint de réagir en augmentant les impôts et en réduisant les dépenses publiques. Britain Stronger in Europe avait aussi sollicité les avis de plusieurs établissements bancaires : la banque HSBC indiquait qu'elle serait contrainte de transférer un millier de postes de Londres vers Paris et, d'après les analystes de Morgan Stanley, le Royaume-Uni perdrait 1 à $1,5 \%$ de croissance (Londres connaîtrait une baisse d'activité de 3 à $5 \%$ ).

19 Les partisans de la sortie de l'Union rejetèrent bien sûr tous ces arguments et mirent en avant les points suivants: le Brexit économiserait 19 milliards de livres par an au Royaume-Uni, les réglementations européennes coûtent près de 33 milliards de livres à l'économie britannique par an, le Royaume-Uni ne dispose que de $8 \%$ des voix au sein de l'Union, le Royaume-Uni a tenté, en vain, à 72 reprises de bloquer des décisions prises par le Conseil ${ }^{21}$. Comment peut-on raisonnablement penser que le citoyen britannique fut à même de se forger une opinion à partir de cette avalanche de considérations économiques plus hermétiques ou absconses les unes que les autres? 


\section{Une incertitude totale} que la grande majorité des économistes se sont révélés incapables de prévoir la crise des subprimes qui a ébranlé l'économie mondiale à partir de 2007. Comme le dit fort justement Daniel Hausman, professeur de philosophie à l'université de WisconsinMadison :

Standard economic theory provides useful tools, but it focuses on a very limited range of causal factors [...] When one recognizes all the other factors that affect economic outcomes, from government policies to the whims of nature, it is easy to see that economists cannot predict the economic future with any precision. ${ }^{22}$

21 Un bref recensement de la rhétorique utilisée pour décrire les conséquences que pourrait avoir le Brexit sur l'économie britannique confirme que le mode qui domina fut celui du conditionnel : «Morgan Stanley warns that Brexit could lead to a recession and a sharper fall in investment, sizeable outflows of capital and labour $"^{23}$ ou encore "A "leave" vote is likely to deter investment in the economy and put the U.K.'s financial services sector at a competitive disadvantage versus other global financial centers ${ }^{24}$ (Standard and Poor's, Britain Stronger in Europe). Il n'est guère surprenant de constater que l'une des stratégies des opposants au Brexit consista à mettre en avant la totale incertitude en la matière. La variété des adjectifs utilisés pour qualifier cette incertitude fut impressionnante: inévitable, importante, significative, massive ou encore considérable. Dans le même ordre d'idée, on notera la récurrence de la notion de risque : "There is a high risk that the UK could vote to leave the European Union with significant economic damages resulting from such a risk scenario " ${ }^{25}$.La rhétorique des opposants au Brexit comporta parfois des métaphores très explicites : "It is like playing Russian roulette with not six bullets in the barrel but at least four $»^{26}$. Un représentant de la Deutsche Bank ${ }^{27}$ alla jusqu'à comparer le Brexit à un voyage au fond d'un terrier. Victor Blank, ancien président de Lloyds Banking Group, estima que la sortie de l'Union serait un désastre ${ }^{28}$. Adam Posen, ancien collaborateur de la Banque d'Angleterre et président d'un club de réflexion à Washington, écrivit que le Brexit reviendrait à se tirer une balle dans le pied (« a horrible self-inflicted wound $»^{29}$ ).

La situation se complique encore davantage quand on ajoutera qu'un nombre non négligeable d'analystes affirmèrent que l'économie britannique bénéficierait d'une sortie de l'Union, à l'image de Patrick Minford de l'Ecole de Commerce de Cardiff qui indiqua que «In the long-term, Brexit will herald a major growth-boosting period " ${ }^{30}$. Les spécialistes les plus éminents proposant des scénarios radicalement différents, voire totalement contradictoires, l'incertitude était donc totale. Comme le résuma prosaïquement le Financial Times dans un article de février 2016: « Economists cannot predict what will happen if Britain leaves the $E U »{ }^{31}$

\section{L'exemple du référendum sur l'indépendance de l'Ecosse}

Le référendum sur le maintien du Royaume-Uni dans l'Union ne fut pas le seul, loin s'en faut, à proposer une question en apparence simple nécessitant des connaissances que la grande majorité des citoyens n'ont pas. Le 18 septembre 2014, les Ecossais eurent à

Revue Française de Civilisation Britannique, XXII-2 | 2017 
répondre à la question suivante : «Should Scotland be an independent country? » La question semble d'une simplicité limpide et l'extraordinaire taux de participation (plus de $84 \%$ ) prouve que les Ecossais se sentirent non seulement concernés mais aussi capables de prendre une décision fondamentale pour l'avenir de leur nation. Les Ecossais prirent la décision de rester au sein du Royaume-Uni, dans une proportion d'environ $55 \%$.

L'une des questions essentielles était de savoir si une Ecosse indépendante se trouverait dans une meilleure situation financière et économique qu'une Ecosse faisant partie du Royaume-Uni. Le gouvernement britannique lui-même reconnut l'importance des enjeux économiques dans le débat: "The economic arguments aren't always easy to follow, but they're vital to understanding the impact of independence, and the effects on our personal finances ${ }^{32}$. Il semble difficile, en effet, de contredire les rédacteurs du texte quand ils soulignent, par le biais d'une belle litote, que les arguments économiques «ne sont pas toujours faciles à suivre ». Les deux camps proposèrent bien sûr des scénarios radicalement différents ; les tenants d'une Ecosse britannique, soutenus pas une multitude d'experts en économie fournissant pléthore de données chiffrées et de statistiques, ne cessèrent d'insister, en utilisant une rhétorique très proche de celle mise en œuvre par les tenants du maintien du Royaume-Uni dans l'Union européenne, sur le risque que représenterait l'indépendance :

The Institute for Fiscal Studies, the Centre for Public Policy for Regions, Citigroup and many other independent experts agree that an independent Scotland would start off in a more challenging financial position than if it remains part of the UK. An independent Scotland is likely to face a higher level of borrowing, because it will have a bigger shortfall between what the Government gets in tax and what it spends on public services. ${ }^{33}$

L'importance fondamentale de la notion de risque dans la décision que prirent les électeurs écossais a été mise en évidence par de nombreux analystes. Ainsi, les chercheurs du Centre on Constitutional Change ${ }^{34}$ ont souligné que pour un grand nombre d'électeurs il est impossible d'envisager des modifications constitutionnelles radicales tant les risques inhérents à une telle décision sont grands. Les deux camps, celui du Leave et celui du Remain, ont abondamment eu recours à la rhétorique du risque, soulignant l'incertitude fiscale d'une Ecosse indépendante ou, au contraire, l'autonomie limitée dont bénéficierait une Ecosse restant au sein du Royaume-Uni. L'étude menée par ces chercheurs conclut en remarquant que le risque joue un rôle bien plus important dans le choix des électeurs que d'autres éléments qui sont présentés comme fondamentaux, tels le genre, l'âge ou même l'identité nationale.

L'une des principales ambitions de l'épais document de 670 pages publié le 25 novembre 2013 par le gouvernement écossais, Scotland's Future, Your Guide to an Independent Scotland, consista donc à convaincre les Ecossais qu'une Ecosse indépendante serait parfaitement viable d'un point de vue économique : «Independence will allow us to use our own resources and shape our own fiscal and economic policies for Scottish needs and circumstances. This will ensure greater economic security and opportunity in the future ${ }^{35}$. Les enjeux économiques étant si difficiles à évaluer, les électeurs écossais prirent vraisemblablement leurs décisions en fonction d'autres facteurs plus empiriques, émotionnels ou subjectifs.

\section{Les limites du référendum}

Il est possible de considérer que ce référendum qui, rappelons-le, tirait avant tout son origine du souhait de David Cameron de donner satisfaction à la frange la plus 
eurosceptique de son parti, ne fut qu'un simulacre de démocratie, dans la mesure où il tendit à faire croire aux citoyens britanniques qu'ils avaient les capacités pour répondre à une question, certes formulée de façon claire, comme il est de coutume dans un exercice de démocratie directe de ce type, mais en fait éminemment complexe. Le volumineux rapport (210 pages) de la commission parlementaire de la Chambre des Lords, publié en février 2010, apporte un éclairage capital sur les implications constitutionnelles du processus référendaire. Le rapport, Referendums in the United Kingdom, qui avait pour ambition d'analyser "le rôle du référendum dans l'expérience constitutionnelle britannique " fait état des débats existant au sein de la classe politique quant à la pertinence des référendums. Dans ce rapport, les douze membres de la commission, après avoir auditionné plus d'une quarantaine d'experts, dressent un état des lieux du référendum et examinent dans le détail les arguments en faveur et en défaveur de cette pratique. Même si le rapport met en évidence le fait que les référendums jouent un rôle pédagogique en provoquant des débats et des discussions qui permettent aux citoyens de prendre une part plus active à la chose publique et, ce faisant, de mieux saisir la portée des enjeux, les référendums, dans un grand nombre de cas, répondent à des considérations purement politiques ou tactiques qui peuvent, en fait, être très éloignées de la question posée :

The balance of the evidence that we have heard leads us to the conclusion that there are significant drawbacks to the use of referendums. In particular, we regret the ad hoc manner in which referendums have been used, often as a tactical device, by the government of the day ${ }^{36}$

La complexité des questions posées lors d'un référendum est l'un des éléments principaux qui fut mis en évidence par certains des experts. Le référendum ne permet pas, dans la mesure où la question posée appelle une réponse par oui ou non, de prendre en compte la variété des questions sous jacentes et ne donne donc qu'une illusion de simplicité. Les parlementaires sont donc les personnes les mieux placées pour se forger une opinion sur des questions complexes :

Whereas elected representatives bring expertise and time to problems that ordinary citizens don't have; they may be more detached and hence objective; and they see the bigger picture of how different issues inter-relate ... a referendum addresses single issues one by one without proper regard to this larger canvass. ${ }^{37}$

Le hiatus entre simplicité et complexité, inhérent à tout référendum, a été souligné lors de débats portant sur d'autres référendums. On évoquera, par exemple, l'un des débats concernant le référendum sur la création de la Greater London Authority qui vit Eric Forth, député conservateur de la circonscription de Bromley and Chislehurst, faire le commentaire suivant :

The debate surely illustrates better than most the folly of referendums as a means of trying to decide what are invariably difficult and complex questions. The decision before us, as it turns out, is just such a difficult and complex question, not the simple-minded question that the Government suggested when they started out on the venture. ${ }^{38}$

Outre le problème de la simplicité trompeuse de la question formulée lors d'un référendum, les experts auditionnés par la commission de la Chambre des Lords remarquèrent que les référendums affaiblissent la représentation parlementaire et diminuent la crédibilité des élus qui sont censés prendre les décisions à la place des citoyens :

Referendums put the people before parliament. The sovereignty of parliament becomes the 
sovereignty of the people (...) Introducing direct democracy into the political system (...) challenges the indirect, representative democracy that has been the essence of UK democracy. ${ }^{39}$

En dépit de ces réserves et de façon quelque peu surprenante, la commission parlementaire reconnut que les référendums pouvaient éventuellement être utilisés pour répondre à des questions d'ordre constitutionnel "fondamentales». Bien que la commission reconnût qu'il est difficile de définir avec précision la notion de questions d'ordre constitutionnel «fondamentales », elle en donna quelques exemples, l'abolition de la monarchie, le maintien dans l'Union européenne, l'abolition d'une des chambres du Parlement, la modification du système électoral ou encore l'adoption d'une constitution écrite.

Les arguments avancés par les partisans et les opposants au Brexit dans la phase finale de la campagne, par les voix de Boris Johnson et de David Cameron, incitèrent sans doute un grand nombre de Britanniques à se déterminer en fonction de considérations émotionnelles ou empiriques. Boris Johnson, tout d'abord: « Napoleon, Hitler, various people tried this [to unify Europe under a single government] out, and it ends tragically. The EU is an attempt to do this by different methods " ${ }^{40}$. David Cameron ensuite: "Can we be so sure that peace and stability on our continent are assured beyond any shadow of doubt? Is that a risk worth taking? I would never be so rash as to make that assumption $»^{41}$. Comparer les dirigeants européens à des dictateurs ou brandir le spectre de la guerre sont deux prises de position tout aussi discutables et critiquables l'une que l'autre. On peut, sans grand risque de se tromper, estimer que les Britanniques firent également leur choix par rapport au « facteur de la peur ». Première peur, la peur de l'autre, en l'occurrence l'immigré, qui eut sans doute une influence déterminante dans la décision que prirent les électeurs. Autre peur, celle consécutive à la menace brandie par George Osborne, le Chancelier de l'Echiquier, qui, quelques jours avant le scrutin, annonça que si le camp du Leave devait l'emporter, le gouvernement serait obligé de procéder à des coupes budgétaires (diminution de $5 \%$ des budgets de la police, des transports et des collectivités locales) et d'augmenter les impôts de près de $2 \%$.

\section{Conclusion}

Le Brexit aura eu un « mérite », cependant, celui de montrer que le Royaume-Uni traverse une véritable crise existentielle, matérialisée par de profondes divisions et lignes de fracture, géographiques, entre Londres ( $60 \%$ pour le Remain) et les comtés du nord (entre 54 et $58 \%$ pour le Leave), nationales, entre l'Ecosse et l'Irlande du Nord (respectivement 62 et $56 \%$ en faveur du Remain) et l'Angleterre (53\% pour le Leave), générationnelles ( $73 \%$ des 18-24 ans votant en faveur du Remain et $60 \%$ des plus de 65 ans votant pour le Leave) et sociales, entre riches et pauvres ( $77 \%$ en faveur du Leave dans les régions aux salaires les plus bas). En vertu des principes fondamentaux qui servent de base à tout régime démocratique, les citoyens choisissent des élus qu'ils estiment avoir les compétences nécessaires pour répondre à des questions complexes. Il est clair que lorsque ces élus s'abaissent à utiliser des arguments ineptes, le citoyen est légitimement en droit de s'interroger sur la pertinence du système. Il n'en reste pas moins que le transfert volontaire de pouvoir est une des pierres angulaires de nos démocraties. Ceci étant, l'exercice référendaire peut avoir toute sa pertinence dans le cadre de questions d'ordre moral ou éthique comme le mariage pour tous, l'avortement ou encore l'euthanasie, où les citoyens sont en mesure de juger par eux-mêmes, mais il 
n'en est pas de même pour des questions techniques d'ordre constitutionnel ou économique pour lesquelles des connaissances ou des compétences spécifiques sont requises, voire indispensables. Lors d'un colloque sur les liens entre référendum et démocratie organisé en novembre 2011, Jean-Marc Sauvé, vice-président du Conseil d'Etat, remarquait que certaines questions pouvaient difficilement être tranchées par le biais du référendum :

La réponse binaire par oui ou par non à des enjeux souvent multiples et complexes peut en outre s'avérer trop simplificatrice et donc inadaptée. Tout choix politique doit, pour être intelligible, être aussi simple et clair que possible, mais beaucoup de choix ne peuvent, sans appauvrissement dangereux de la prise de décision, se réduire à deux options antagonistes. ${ }^{42}$

La démocratie participative, permet, en théorie, de donner la parole directement au peuple et le référendum constitue un des moyens par lesquels une démocratie peut affirmer sa vitalité. Il n'en reste pas moins que cet outil a malheureusement ses limites et l'instrumentaliser à des fins politiques, comme le fit le Premier ministre britannique, constitue une démarche qui affaiblit les fondements démocratiques. Il est fort à craindre que David Cameron, tout comme Neville Chamberlain ou Anthony Eden, entrera dans l'histoire comme l'auteur d'une calamiteus e erreur pour l'avenir de son pays.

Christian AUER, Agrégé de l'Université, Professeur Emérite à l'Université de Strasbourg, est spécialiste de civilisation britannique. Il travaille principalement sur les aspects économiques, politiques, sociaux et culturels de l'Ecosse au XIX siècle. Il est l'auteur de nombreux articles et de plusieurs ouvrages dont Scotland and the Scots 1707-2007 A Reader (Strasbourg : Presses Universitaires de Strasbourg, 2013) et Luttes et résistances des femmes écossaises, 1838-1915 (Paris, L'Harmattan, Collection des idées et des femmes, 2013).

\section{BIBLIOGRAPHIE}

BOGDANOR Vernon, The People and the Party System: The Referendum and Electoral Reform in British Politics, Cambridge: Cambridge University Press, 1981.

D’HELLENCOURT Bernard et SCHNAPPER Pauline (dir.), « La pratique référendaire dans les îles britanniques », Revue Française de Civilisation Britannique, Hors série n² 2, mai 2009.

DUCLOS Nathalie (dir.), «Le référendum sur l'indépendance écossaise du 18 septembre 2014 », Revue Française de Civilisation Britannique, vol. XX-2, 2015.

GIBBINS Justin, Britain, Europe and National Identity: Self and Other in International Relations, Basingstoke: Palgrave Macmillan, 2014.

HENDERSON Ailsa, DELANEY Liam, LINEIRA Robert, « Risk, Uncertainty and Vote choice in the Scottish Referendum », Centre on Constitutional Change, 15 août 2014.

MARSHALL Geoffrey, "The Referendum: What, When and How? ", Parliamentary Affairs, vol. 50, n 2, 1997, p. 307-313, 307. 
SANDBROOK Dominic, Seasons in the Sun The Battle for Britain 1974-1979 (2012), Londres: Penguin

Books, 2013.

THATCHER Margaret, The Path to Power, Londres: Harper Collins, 1995.

\section{NOTES}

1. Global Counsel, Brexit: The Impact on the UK and the EU, June 2015, https://www.globalcounsel.co.uk/sites/default/files/special- $\quad$ reports/downloads/Global\% 20Counsel_Impact_of_Brexit.pdf.

2. 67,2 \% des citoyens se prononcèrent en faveur du maintien : "Do you think the United Kingdom should stay in the European Community (the Common Market)? »

3. $67,9 \%$ des citoyens se prononcèrent en faveur du maintien du système électoral uninominal à un tour : "At present, the UK uses the "first past the post" system to elect MPs to the House of Commons. Should the "alternative vote" system be used instead?"

4. Vernon Bogdanor, The People and the Party System: The Referendum and Electoral Reform in British Politics, Cambridge: Cambridge University Press, 1981, p. 1.

5. Geoffrey Marshall, « The Referendum: What, When and How? », Parliamentary Affairs, vol. 50, n 2, 1997, p. 307-313, p. 307.

6. January 1910 Conservative Party General Election Manifesto, Arthur Balfour's Election Address.

7. Margaret Thatcher, The Path to Power, Londres: Harper Collins, 1995, p. 402.

8. Margaret Thatcher, Hansard Parliamentary Debates, 11 mars 1975, vol. 888, col. 314.

9. Margaret Thatcher, Hansard Parliamentary Debates, 11 mars 1975, vol. 888, col. 310.

10. Let us work together - Labour's way out of the crisis, 1974.

11. Cité dans Justin Gibbins, Britain, Europe and National Identity: Self and Other in International Relations, Basingstoke: Palgrave Macmillan, 2014, p. 56.

12. "A new settlement for the United Kingdom in a reformed European Union, " David's Cameron letter to Donald Tusk, 10 November 2015.

13. "The United Kingdom and the European Union », European Council, Brussels, 19 février 2016, EUCO $1 / 16$ CO EUR 1 CONCL.

14. «A new settlement for the United Kingdom in a reformed European Union, » David's Cameron letter to Donald Tusk, 10 November 2015.

15. «Ever Closer Union », London School of Economics European Institute, Report of the hearing held on 15th April, 2016.

16. «The United Kingdom and the European Union », European Council, Brussels, 19 février 2016, EUCO 1/16 CO EUR 1 CONCL.

17. European Council Declaration on Competitiveness, 2 février 2016 (http://www.politico.eu/ wp-content/uploads/2016/02/Delegations-will-find-attached-a-revised-version-of-the-draft-

European-Council-Declaration-on-competitiveness.pdf).

18. "Martin Schulz: 'L'Europe est une promesse qui n'a pas été tenue' », Le Soir, 5 mai 2016.

19. «Staying in EU 'exposes UK to terror risk', says Iain Duncan Smith », 21 février 2016, http:// www.bbc.com/news/uk-politics-eu-referendum-35624409.

20. «EU referendum: What are the pros and cons of Brexit?», 27 juin 2016, http:// www.theweek.co.uk/eu-referendum.

21. Vote Leave, Take Control, www.voteleavetakecontrol.org.

22. Gary Gutting, «What Economics Can (and Can't) Do », The New York Times, 14 juillet 2015.

23. Britain Stronger in Europe, " 100 Times the Leave Campaigns Lost the Economic Argument", $\mathrm{p} 4$, https://d3n8a8pro7vhmx.cloudfront.net/in/pages/1539/attachments/ 
original/1457978567/100_Ways_Leave_Campaigners_Have_Lost_The_Economic_Argument.pdf? 1457978567.

24. Britain Stronger in Europe, "100 Times the Leave Campaigns Lost the Economic Argument ", p. 12.

25. M. Leglan, Société Générale, 4 janvier 2016, Britain Stronger in Europe, « 100 Times the Leave Campaigns Lost the Economic Argument ", p. 14.

26. Directeur Général d'Axa, 31 janvier 2016, Britain Stronger in Europe, " 100 Times the Leave Campaigns Lost the Economic Argument », p. 19.

27. Deutsche Bank, $1^{\mathrm{er}}$ mars 2016, Britain Stronger in Europe, « 100 Times the Leave Campaigns Lost the Economic Argument ", p. 29.

28. Victor Blank, 30 décembre 2015, Britain Stronger in Europe, « 100 Times the Leave Campaigns Lost the Economic Argument », p. 5.

29. Ben Chu, "'Self-inflicted wound' of EU vote could drive UK back into recession, says Adam Posen », The Independent, 22 janvier 2016.

30. Chris Giles, "What are the economic consequences of Brexit? », Financial Times, 22 février 2016.

31. Ibid.

32. Scotland's Future, Your Guide to an Independent Scotland, 26 novembre 2013, GOV. UK, Scotland Office (https://www.gov.uk/government/publications/scottish-independence-referendumpersonal-finance/scottish-independence-referendum-personal-finance.)

33. Ibid.

34. Ailsa Henderson, Liam Delaney, Robert Lineira, « Risk, Uncertainty and Vote choice in the Scottish Referendum », Centre on Constitutional Change, 15 août 2014.

35. Scotland's Future, Your Guide to an Independent Scotland, 26 novembre 2013, p. 85.

36. Referendums in the United Kingdom, House of Lords, Select Committee on the Constitution, 12th Report of Session 2009-10, p. 20.

37. Stephen Tierney, Université d'Edimbourg, Referendums in the United Kingdom, House of Lords, Select Committee on the Constitution, 12th Report of Session 2009-10, p. 48.

38. Eric Forth, Hansard Parliamentary Debates, 11 février 1998, vol. 306, col. 500.

39. Peter Browning, Université de Drury, Missouri, Referendums in the United Kingdom, House of Lords, Select Committee on the Constitution, 12th Report of Session 2009-10, p. 112-3.

40. Tim Ross, « Boris Johnson: The EU wants a superstate, just as Hitler did », The Daily Telegraph, 15 mai 2016.

41. Steven Swinford, "David Cameron: Brexit could lead to Europe descending into war », The Daily Telegraph, 9 mai 2016.

42. Jean-Marc Sauvé, "Référendum et démocratie ", Colloque "Théorie et pratiques du référendum ", organisé par la Société de législation comparée, 4 novembre 2011 (http:// www.conseil-etat.fr/Actualites/Discours-Interventions/Referendum-et-democratie)

\section{RÉSUMÉS}

Lors du référendum sur le maintien du Royaume-Uni dans l'Union européenne qui s'est tenu le 23 juin 2016, les Britanniques ont pris une décision fondamentale non seulement pour l'avenir de leur pays, mais également pour celui de l'Union toute entière. Outre les questions telles la place 
du Royaume-Uni dans l'Europe et dans le monde, la souveraineté nationale, l'immigration ou encore la sécurité, les aspects d'ordre économique constituèrent sans nul doute un des enjeux majeurs. Les Britanniques furent en effet amenés à imaginer les conséquences d'un éventuel Brexit sur le commerce, l'investissement, le marché de l'emploi ou encore la libre circulation des biens, des personnes, des services et des capitaux. Une majorité d'économistes furent favorables à un maintien dans l'Union mais un nombre non négligeable d'analystes affirmèrent que l'économie britannique bénéficierait d'une sortie de l'Union. Comme l'a remarquablement (et prosaïquement) résumé le Financial Times dans un article du 22 février 2016: « Economists cannot predict what will happen if Britain leaves the $E U »$. Il est donc légitime de se demander comment les citoyens britanniques furent en mesure de faire un choix éclairé alors que les spécialistes les plus éminents proposèrent des scénarios totalement contradictoires. Les électeurs se déterminèrent vraisemblablement en fonction de facteurs plus empiriques, émotionnels ou subjectifs. Ce référendum ne fut donc qu'un simulacre de démocratie, dans la mesure où il tendit à faire croire aux citoyens britanniques qu'ils avaient les capacités et les compétences pour répondre à une question, certes formulée de façon claire, comme il est de coutume dans un exercice de démocratie directe de ce type, mais en fait éminemment complexe.

With the referendum on Brexit of June 232016 the British took a fundamental decision not only for the future of their country but also for the future of the entire European Union. As well as issues such as the place of the United Kingdom in Europe and in the world, national sovereignty, immigration or security, economic aspects - commerce, investments, the labour market or the free movement of goods, persons, services and capital - were undoubtedly fundamental. Most economists such as Jonathan Portes from the National Institute of Economic and Social Research who said that Brexit would be "a bloody nightmare" were against Brexit. Some analysts, though, contended that the British economy would benefit from Brexit. As was remarkably summed up by an article of the Financial Times published on February 22 2016: "Economists cannot predict what will happen if Britain leaves the EU". It is thus legitimate to wonder whether the British were in a position to make a clear choice. Empirical, emotional or subjective factors probably played an important part in the British decision. It should be remembered that David Cameron decided to call a referendum on Britain's EU membership after bowing to pressure from the Eurosceptic wing of the Conservative Party. Thus the referendum was a mockery of democracy as the British were misled into believing that they could answer a very simple question, which was in fact highly complex.

\section{INDEX}

Keywords : Brexit, European Union, referendum, representative democracy, participatory democracy

Mots-clés : Brexit, Union européenne, référendum, démocratie représentative, démocratie participative

\section{AUTEUR}

\section{CHRISTIAN AUER}

Université de Strasbourg 